\title{
SUPERSONIC QUADRUPOLE NOISE THEORY FOR HIGH-SPEED HELICOPTER ROTORS*
}

\author{
F. Farassat and Kenneth S. Brentner \\ Fluid Mechanics and Acoustics Division, NASA Langley Research Center \\ Hampton, Virginia 23681-0001, U.S.A.
}

\begin{abstract}
SUMMARY
High-speed helicopter rotor impulsive noise prediction is an important problem of aeroacoustics. The deterministic quadrupoles have been shown to contribute significantly to high-speed impulsive (HSI) noise of rotors, particularly when the phenomenon of delocalization occurs. At high rotor-tip speeds, some of the quadrupole sources lie outside the sonic circle and move at supersonic speed. Brentner has given a formulation suitable for efficient prediction of quadrupole noise inside the sonic circle. In this paper, we give a simple formulation based on the acoustic analogy that is valid for both subsonic and supersonic quadrupole noise prediction. Like the formulation of Brentner, the model is exact for an observer in the far field and in the rotor plane and is approximate elsewhere. We give the full analytic derivation of this formulation in the paper. We present the method of implementation on a computer for supersonic quadrupoles using marching cubes for constructing the influence surface ( $\Sigma$ surface) of an observer spacetime variable $(\mathbf{x}, t)$. We then present several examples of noise prediction for both subsonic and supersonic quadrupoles. It is shown that in the case of transonic flow over rotor blades, the inclusion of the supersonic quadrupoles improves the prediction of the acoustic pressure signature. We show the equivalence of the new formulation to that of Brentner for subsonic quadrupoles. It is shown that the regions of high quadrupole source strength are primarily produced by the shock surface and the flow over the leading edge of the rotor. The primary role of the supersonic quadrupoles is to increase the width of a strong acoustic signal.
\end{abstract}

\section{INTRODUCTION}

High-speed flight of helicopters has been an illusive goal because of the rapid increase in rotor noise and vibration. Noise and vibration increase dramatically because of the increasing disparity between the fluid velocity over the advancing and the retreating rotor blades. When the supersonic flow region on the advancing rotor blade extends off the blade into the far field (a phenomenon known as delocalization [1]), high-speed impulsive (HSI) noise becomes dominant over all the other rotor noise sources. For this reason, the efficient prediction of the HSI noise of helicopter rotors is currently an important problem of aeroacoustics. The cause of this noise has been identified since the late 1970's [1,2] as the deterministic quadrupoles in the vicinity of the rotor, and, in the case of delocalization, beyond the sonic circle and the blade tip. Many schemes have been proposed by researchers based on the acoustic analogy [3-9] and the Kirchhoff method [10, 11]. At present, most of these schemes are limited to subsonic quadrupole source motion, however, very recently several methods have been proposed for the prediction of noise from supersonic sonic sources [12-15]. In the case of subsonic quadrupole noise prediction, a method based on a formulation by Brentner, formulation Q1A, exists [7] which is exact for an in-plane, far-field observer but is approximate elsewhere. This method has been implemented in the code WOPWOP $+[6,7]$ and has been shown to be highly efficient and robust. There is a need for an efficient and robust method of prediction of the supersonic quadrupole noise. We present a new formulation — based on the same model used by Brentner - that is valid for both subsonic and supersonic quadrupole noise prediction.

The derivation of a supersonic quadrupole formulation, which we call formulation Q2, is the main result of this paper. In Section 2 we start with an exact solution of the wave equation for quadrupole sources of the Ffowcs Williams-Hawkings (FW-H) equation given by Farassat and Brentner [16]. In this solution, volume integrals involving the quadrupole sources are only differentiated with respect to observer time. We write

*Presented at the American Helicopter Society Technical Specialists' Meeting for Rotorcraft Acoustics and Aerodynamics, Williamsburg, Virginia, October 28 - 30, 1997. Published in Journal of Sound and Vibration. 
these volume integrals in terms of a surface integral over the collapsing sphere and a source time integral. For an observer in the rotor plane and in the far field, the collapsing sphere is approximated as a right circular cylinder normal to the rotor disc and the quadrupole source strength is integrated along lines normal to the rotor disc and treated as sources on the rotor disc. We then hypothesize that the quadrupole noise everywhere can be predicted using these surface sources. This hypothesis has been validated by Brentner and Holland [6]. The idea of approximating the volume (quadrupole) sources with equivalent surface sources was originally proposed and numerically implemented by Yu et al. [1] for the far-field solution of the FW-H equation. Later this idea was also implemented by Schultz and Splettstoesser [3], Brentner and Holland [6], and Brentner [7]. Our main contribution has been to use this idea in obtaining closed-form solutions of the same equation, formulations Q1A and Q2, which seem to result in more efficient prediction of HSI noise. The new formulation (Q2) presented here is very simple and unlike formulation Q1A is valid for both subsonic and supersonic quadrupoles. Hence we can directly compare quadrupole noise predictions from both formulations in the subsonic case.

In Section 3 we discuss how formulation Q2 is implemented in a new testbed code called WOPWOP2+. The quadrupole sources beyond the sonic circle can have multiple emission times and the usual solution of the wave equation for subsonic surface sources (e.g., formulations 1, 1A, and Q1A) will have a singularity known as the Doppler singularity. To avoid this Doppler singularity, it is necessary to use a $\Sigma$-surface formulation $[17,18]$. The method of construction of the $\Sigma$ surface used in WOPWOP2+ is known as marching cubes [19] — an algorithm originally developed for computer graphics [20].

Some examples of HSI noise prediction for a hovering rotor is presented in Section 4. First we present a comparison of the results of noise prediction for subsonic quadrupoles based on formulations Q1A and Q2. It is shown that the results agree well with each other. A study of the surface source strength (i.e., the integral of quadrupole source strength along the line normal to rotor disc) shows that the primary contributions to the quadrupole noise come from the shock surfaces on and beyond the blade and the flow over the leading edge of the blade. It is also shown that the inclusion of the quadrupole sources beyond the sonic circle improves the prediction of the width of the main pulse and the shape of the acoustic pressure signature and agreement with experimental data. Finally, we demonstrate the robustness of the formulation by performing predictions for out-of-plane and near-field observers. Concluding remarks follow in Section 5.

\section{FORMULATION AND SOLUTION OF THE PROBLEM}

We begin with the solution of the following wave equation for quadrupole noise radiation from the Ffowcs Williams-Hawkings equation:

$$
\square^{2} p_{Q}^{\prime}(\mathbf{x}, t)=\frac{\bar{\partial}^{2}}{\partial x_{i} \partial x_{j}}\left[T_{i j} H(f)\right]
$$

where the bar over the partial derivative operator indicates generalized differentiation, $T_{i j}$ is the Lighthill stress tensor, $H(f)$ is the Heaviside function, and $f=0$ describes the blade surface $(f>0$ outside the blade). The solution for this equation was given by Farassat and Brentner [16] as follows:

$$
\begin{aligned}
4 \pi p_{Q}^{\prime}(\mathbf{x}, t) & =\frac{1}{c} \frac{\partial^{2}}{\partial t^{2}} \int_{-\infty}^{t} \int_{f>0} \frac{T_{r r}}{r} d \Omega d \tau \\
& +\frac{\partial}{\partial t} \int_{-\infty}^{t} \int_{f>0} \frac{3 T_{r r}-T_{i i}}{r^{2}} d \Omega d \tau \\
& +c \int_{-\infty}^{t} \int_{f>0} \frac{3 T_{r r}-T_{i i}}{r^{3}} d \Omega d \tau
\end{aligned}
$$

where the quantity $T_{r r}$ is the double contraction $T_{i j} \hat{r}_{i} \hat{r}_{j}$, and $\hat{r}_{i}$ are the components of the unit vector in the radiation direction. In addition, $d \Omega$ is an element of the collapsing sphere surface $g=0$. We now assume that the observer is in the far field and on the rotor plane. The part of the collapsing sphere intersecting 


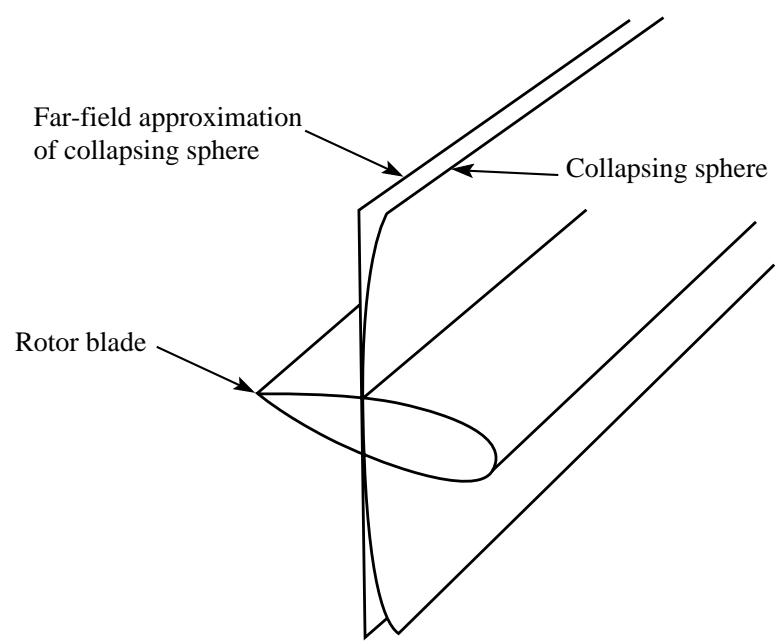

Figure 1. The actual and approximate collapsing sphere surfaces in the vicinity of the rotor blade.

the source region near the rotor blade can be approximated by a right circular cylinder normal to the rotor plane. This is shown in figure 1.

Let us assume that the rotor is nominally in the $y_{1} y_{2}$-plane and $y_{3}$ is, therefore, perpendicular to this plane (i.e., the rotor tip-path plane). We integrate the inner integrals of equation (2) with respect to $y_{3}$ over the approximate collapsing sphere surface. Let us define

$$
Q_{i j}\left(y_{1}, y_{2}, \tau\right)=\int_{-\infty}^{\infty} T_{i j} d y_{3}
$$

and use the relation

$$
d \Sigma=c d \tau d,
$$

where $d$, is the length of an element of the curve defined by the intersection of the collapsing sphere with the rotor disc. Equation (2) can now be written as

$$
\begin{aligned}
4 \pi p_{Q}^{\prime}(\mathbf{x}, t) & =\frac{1}{c^{2}} \frac{\partial^{2}}{\partial t^{2}} \int \frac{1}{r}\left[Q_{r r}\right]_{r e t} d \Sigma \\
& +\frac{1}{c} \frac{\partial}{\partial t} \int \frac{1}{r^{2}}\left[3 Q_{r r}-Q_{i i}\right]_{r e t} d \Sigma \\
& +\int \frac{1}{r^{3}}\left[3 Q_{r r}-Q_{i i}\right]_{r e t} d \Sigma
\end{aligned}
$$

where the subscript ret indicates the integrand is evaluated at the retarded time. The integrals in equation (5) are all over the entire $y_{1} y_{2}$-plane. This fact allows us to bring the observer-time derivatives inside the integrals without worrying about the limits of integration.

The next step is the most crucial in the derivation of the final result. We note that $\partial / \partial t=\partial /\left.\partial t\right|_{\mathbf{x}}$ meaning $\mathbf{x}$ in the frame fixed to the undisturbed medium is kept fixed in this differentiation. Also note that the tensor $\mathbf{Q}$ with components $Q_{i j}$ is defined in the $\mathbf{x}$ frame of reference, hence all velocity terms in $Q_{i j}(\mathbf{x}, t)$ are specified with respect to the frame fixed to the undisturbed medium. However, if we use a change of coordinates $(\mathbf{x}, t) \rightarrow(\boldsymbol{\eta}, \tau)$, where the $\boldsymbol{\eta}$-frame is aligned with the rotor blade (i.e., by coordinate rotation 
and translation), we have

$$
\begin{aligned}
\left.\frac{\partial\left[Q_{i j}\right]_{r e t}}{\partial t}\right|_{\mathbf{x}} & =\left[\left.\frac{\partial}{\partial \tau}\right|_{\mathbf{x}} Q_{i j}\right]_{r e t} \\
& =\left[\left.\frac{\partial Q_{i j}}{\partial \tau}\right|_{\boldsymbol{\eta}}-\mathbf{V} \cdot \nabla_{\boldsymbol{\eta}} Q_{i j}\right]_{r e t} \\
& \equiv\left[L_{\tau} Q_{i j}\right]_{r e t}
\end{aligned}
$$

where $\boldsymbol{\eta}$ is the position vector in the rotating frame and $\tau$ is the source time. Here $\mathbf{V}=\partial \boldsymbol{\eta} / \partial \tau$ is the velocity of the point $\boldsymbol{\eta}$ specified in the frame fixed to the undisturbed medium. We note that $\mathbf{V}$ is in the rotor plane. It is important to recognize that when we refer to $\left.Q_{i j}\right|_{\eta}$ we really mean that the components of the tensor $Q_{i j}$ represented in coordinates that are instantaneously aligned with the rotating frame. Thus equation (6) provides the time derivative of $\left.Q_{i j}\right|_{\mathbf{x}}$ in the stationary frame in terms of $\left.Q_{i j}\right|_{\boldsymbol{\eta}}$ which is specified in the coordinates of the moving frame. Using this operator notation in equation (5), we get

$$
\begin{aligned}
4 \pi p_{Q}^{\prime}(\mathbf{x}, t) & =\frac{1}{c^{2}} \int \frac{1}{r} \hat{r}_{i} \hat{r}_{j}\left[L_{\tau}^{2} Q_{i j}\right]_{r e t} d \Sigma \\
& +\frac{1}{c} \int \frac{1}{r^{2}}\left[3 \hat{r}_{i} \hat{r}_{j} L_{\tau} Q_{i j}-L_{\tau} Q_{i i}\right]_{r e t} d \Sigma \\
& +\int \frac{1}{r^{3}}\left[3 Q_{r r}-Q_{i i}\right]_{r e t} d \Sigma .
\end{aligned}
$$

Notice that the operator $L_{\tau}$ operates on $Q_{i j}$ only because $\hat{r}_{i}$ and $\hat{r}_{j}$ do not depend upon $t$ or $\tau$. To write this equation in final form we express $\mathbf{V}(\boldsymbol{\eta}, \tau)$ as follows:

$$
\mathbf{V}=\mathbf{V}_{F}+\boldsymbol{\omega} \times \boldsymbol{\eta}
$$

where $\mathbf{V}_{F}$ is the forward velocity of the rotor, $\boldsymbol{\omega}$ is the angular velocity of the rotor, and $\boldsymbol{\eta}$ is the position vector of the source $Q_{i j}$ in the rotor plane with the origin at the rotor center. We assume that both $\mathbf{V}_{F}$ and $\boldsymbol{\omega}$ are time independent and we note that $\omega=|\boldsymbol{\omega}|$. From equation (8), we have

$$
\begin{aligned}
\left.\frac{\partial \mathbf{V}}{\partial \tau}\right|_{\eta} & =\boldsymbol{\omega} \times(\boldsymbol{\omega} \times \boldsymbol{\eta}) \\
& =-\omega^{2} \boldsymbol{\eta} .
\end{aligned}
$$

Thus, we can express $L_{\tau}^{2}$ as follows:

$$
\begin{aligned}
L_{\tau}^{2}= & \left(\left.\frac{\partial}{\partial \tau}\right|_{\boldsymbol{\eta}}-\mathbf{V} \cdot \nabla\right)\left(\left.\frac{\partial}{\partial \tau}\right|_{\boldsymbol{\eta}}-\mathbf{V} \cdot \nabla\right) \\
= & \left.\frac{\partial^{2}}{\partial \tau^{2}}\right|_{\boldsymbol{\eta}}-\left.\mathbf{V} \cdot \nabla \frac{\partial}{\partial \tau}\right|_{\boldsymbol{\eta}}-\left.\frac{\partial}{\partial \tau}\right|_{\boldsymbol{\eta}}(\mathbf{V} \cdot \nabla) \\
& +\mathbf{V} \cdot \nabla(\mathbf{V} \cdot \nabla)
\end{aligned}
$$

All the gradients are with respect to $\eta$. We have

$$
\frac{\partial}{\partial \tau}(\mathbf{V} \cdot \nabla)=-\omega^{2} \eta \frac{\partial}{\partial \eta}+\mathbf{V} \cdot \nabla \frac{\partial}{\partial \tau}
$$

where $\partial / \partial \eta$ is the directional derivative in the $\boldsymbol{\eta}$ (radial direction) and $\eta=|\boldsymbol{\eta}|$. We also have

$$
\begin{aligned}
\mathbf{V} \cdot \nabla(\mathbf{V} \cdot \nabla)= & (\boldsymbol{\omega} \times \mathbf{V}) \cdot \nabla+\left(\mathbf{V} \cdot \nabla^{\prime}\right)^{2} \\
= & \left(\boldsymbol{\omega} \times \mathbf{V}_{F}\right) \cdot \nabla-\omega^{2} \eta \frac{\partial}{\partial \eta} \\
& +\left(\mathbf{V} \cdot \nabla^{\prime}\right)^{2}
\end{aligned}
$$

where $\nabla^{\prime}$ does not operate on $\mathbf{V}$, i.e., we define

$$
\left(\mathbf{V} \cdot \nabla^{\prime}\right)^{2} \equiv V_{1}^{2} \frac{\partial^{2}}{\partial \eta_{1}^{2}}+2 V_{1} V_{2} \frac{\partial^{2}}{\partial \eta_{1} \partial \eta_{2}}+V_{2}^{2} \frac{\partial^{2}}{\partial \eta_{2}^{2}}
$$


and $\mathbf{V}=\left(V_{1}, V_{2}\right)$. Therefore, $L_{\tau}^{2}$ can be written as

$$
\begin{aligned}
L_{\tau}^{2}= & \left.\frac{\partial^{2}}{\partial \tau^{2}}\right|_{\boldsymbol{\eta}}-\left.2 \mathbf{V} \cdot \nabla \frac{\partial}{\partial \tau}\right|_{\boldsymbol{\eta}}+\left(\mathbf{V} \cdot \nabla^{\prime}\right)^{2} \\
& +\left(\boldsymbol{\omega} \times \mathbf{V}_{F}\right) \cdot \nabla
\end{aligned}
$$

When this expression is used in equation (7), we get a singularity free expression for supersonic quadrupole noise prediction. We note that equation (7) has second space and time derivatives of $Q_{i j}$ as well as first space derivatives in the $\eta_{1} \eta_{2}$-plane (the rotor plane). These quantities are available in the CFD postprocessor that is used to compute $Q_{i j}$ for acoustic calculations. We will refer to equation (7) as formulation Q2. As it stands, formulation Q2 is valid for subsonic and supersonic quadrupole noise prediction for helicopter rotors in hover or forward flight. Note that this equation is very simple and has no singularities. We have assumed that the shocks on the blades are smeared over one or more grid cells and that $Q_{i j}$ has continuous second derivatives over the rotor plane (although the magnitude of the second derivative can be very high at the foot of the shocks). These assumptions are generally satisfied in CFD calculations for helicopter rotor aerodynamics.

\subsection{ANALYSIS OF MAIN RESULT}

We will now do an order of magnitude study of the far-field term of our main result, equation (7). We can draw very useful conclusions from such a study as will be shown below. We know that the peak of directivity of quadrupole noise is in the rotor plane with the observer ahead of the helicopter. Let us put the observer in such a location in the far field. Then in the frame fixed to the undisturbed medium (where $x_{1}$-axis is the flight direction and the $x_{3}$-axis is normal to the rotor plane), the components of the unit radiation vector can be approximated as $\hat{\mathbf{r}}=(1,0,0)$. The major contribution to the far-field quadrupole noise comes from $Q_{11}$ which we will look at closely below.

The numerator of the integrand of the far-field term is

$$
\begin{aligned}
L_{\tau}^{2} Q_{11}= & \left.\frac{\partial^{2} Q_{11}}{\partial \tau^{2}}\right|_{\boldsymbol{\eta}}-\left.2 \mathbf{V} \cdot \nabla \frac{\partial Q_{11}}{\partial \tau}\right|_{\boldsymbol{\eta}}+\left(\mathbf{V} \cdot \nabla^{\prime}\right)^{2} Q_{11} \\
& +\left(\boldsymbol{\omega} \times \mathbf{V}_{F}\right) \cdot \nabla Q_{11}
\end{aligned}
$$

We can now estimate the order of magnitude of each term in equation (15) as follows. Let the advancing tip speed be denoted by $V_{A T}$. Then, we see that

$$
\begin{aligned}
\left.\frac{\partial^{2} Q_{11}}{\partial \tau^{2}}\right|_{\eta} & \sim \omega^{2} Q_{11} \\
\left.\mathbf{V} \cdot \nabla \frac{\partial Q_{11}}{\partial \tau}\right|_{\eta} & \sim \omega V_{A T} \frac{\partial Q_{11}}{\partial \eta_{1}} \\
\left(\mathbf{V} \cdot \nabla^{\prime}\right)^{2} Q_{11} & \sim V_{A T}^{2} \frac{\partial^{2} Q_{11}}{\partial \eta_{1}^{2}} \\
\left(\boldsymbol{\omega} \times \mathbf{V}_{F}\right) \cdot \nabla Q_{11} & \sim \omega V_{F} \frac{\partial Q_{11}}{\partial \eta_{1}}
\end{aligned}
$$

In these equations, the derivative $\partial / \partial \eta_{1}$ is the directional derivative in the chordwise direction. Note that for a hovering rotor $\partial Q_{11} / \partial \tau, \partial^{2} Q_{11} / \partial \tau^{2}$, and $V_{F}$ are all zero, therefore the only remaining component is $\left(\mathbf{V} \cdot \nabla^{\prime}\right)^{2} Q_{11}$. Since $\omega$, in general, is small for helicopter rotors, we can see that the dominant term in forward flight is also most likely the term $\left(\mathbf{V} \cdot \nabla^{\prime}\right)^{2} Q_{11}$. The right side of equation (16c) can be further estimated as

$$
V_{A T}^{2} \frac{\partial^{2} Q_{11}}{\partial \eta_{1}^{2}} \sim \frac{V_{A T}^{2} Q_{11}}{\left(\Delta \eta_{1}\right)^{2}}
$$

where $\Delta \eta_{1}$ is the chordwise scale over which significant change in $Q_{11}$ occurs. Significant changes of $Q_{11}$ occur near both the leading edge stagnation point and the base of the shock. Hence we suspect that the dominant sources of quadrupole noise will be located at these locations $\left(\Delta \eta_{1} \sim L E\right.$ radius and $\Delta \eta_{1} \sim$ width of the projection of the shock surface in the rotor plane). These conclusions are verified by the computation 


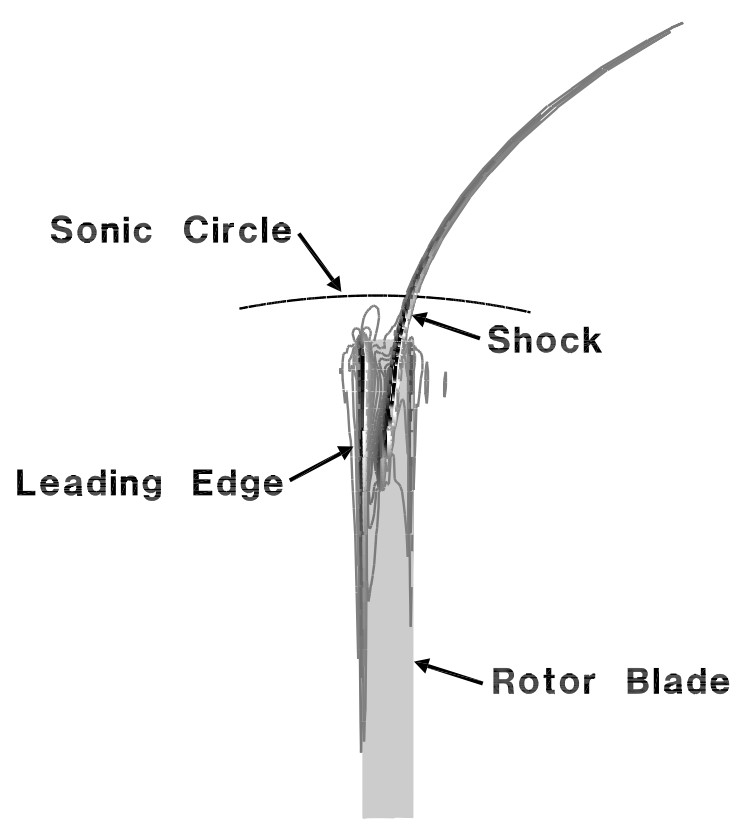

Figure 2. Contours of $L_{\tau}^{2} Q_{11}$ in the vicinity of a hovering UH-1H rotor, $M_{H}=0.925$.

of $L_{\tau}^{2} Q_{11}$ for a hovering $\mathrm{UH}-1 \mathrm{H}$ model rotor blade at tip Mach number 0.925 shown in figure 2 . It is apparent in the figure that the primary source of HSI noise is the shock wave (as proposed by Farassat and his colleagues - see references 16,21-23) and the flow over the leading edge of the blade. The significance of the quadrupole source in the leading edge region has not been widely recognized in previous work, however, it has been found here to be of comparable amplitude to the quadrupole source strength in the shock region.

\section{NUMERICAL IMPLEMENTATION}

A new code, called WOPWOP2+, is used to demonstrate the utility of formulation Q2. WOPWOP2+ differs significantly from WOPWOP $+[6,7]$ in that it uses a $\Sigma$-surface formulation to compute thickness and loading noise, as well as the quadrupole noise. The construction of the $\Sigma$ surface and subsequent integration over the $\Sigma$ surface is performed using the method of marching cubes integration developed by Brentner [19]. The numerical calculation of quadrupole noise has been divided into two stages: a preprocessing stage in which the integration of the Lighthill stress tensor in the normal direction, indicated in equation (3), is carried out, and an evaluation stage in which the quadrupole contribution to the acoustic pressure specified in equation (7) is determined. Both the preprocessor and the acoustic calculation are described briefly in this section. More information on the preprocessor, which is the same preprocessor that is used by WOPWOP+, can be found in reference 6 .

\subsection{PREPROCESSOR}

Although the evaluation of $Q_{i j}$ can be performed independently of observer position and retarded time, the preprocessor must read in the CFD solution, interpolate the solution at the necessary quadrature locations, and then perform the numerical quadrature in the direction normal to the rotor disc. The preprocessor needs knowledge of both the CFD grid topology and the solution format. In the implementation used for this work, the interpolation of the CFD data is two dimensional and is done one radial station at a time. For a given radial station, data are interpolated to quadrature points needed for composite Gauss-Legendre integration, on lines normal to the rotor plane. The lines are uniformly distributed in the chordwise direction. A twodimensional linear least-squares interpolation is used to interpolate the density, momentum, and energy at each quadrature point. The Lighthill stress tensor $T_{i j}$ is evaluated with the interpolated data. The value of $Q_{i j}$ on the rotor disc is determined at each chordwise location before moving to the next radial station. The results are stored for the acoustic calculation stage. The CFD data used for the noise prediction [24] was 


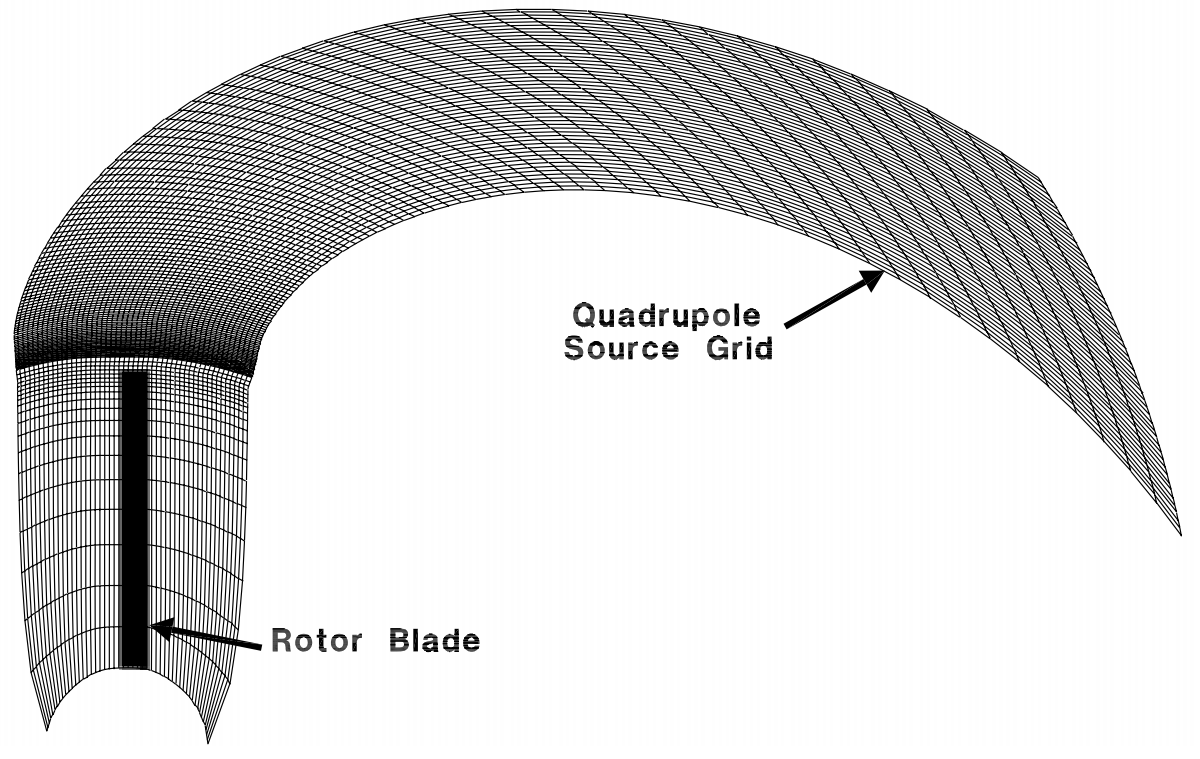

Figure 3. Typical quadrupole grid used for WOPWOP2+ calculations for hovering UH-1H rotor. Note that only every 4 th grid line is shown in the chordwise direction.

given on a grid with rather coarse radial resolution beyond the blade tip. The quadrupole source strength radial resolution off the blade tip was increased five fold by utilizing 6th order polynominal interpolation along radial grid lines for both the location and strength of the quadrupole data. A typical grid generated by the quadrupole preprocessor is shown in figure 3 .

\subsection{WOPWOP2+}

The primary function of the WOPWOP $2+$ code is to perform the integration indicated in equation (7) numerically. Although the integration is over the entire rotor disc plane, in practice the source strength is zero over a large part of the plane; hence, the quadrupole integration is only performed near the rotor blade (see figure 3). The main difficulty in the numerical evaluation of equation (7) is the construction of the $\Sigma$ surface for the portion of the plane in which the source strength is nonzero. The $\Sigma$ surface is the collection of points in space-time that emit signals that reach the observer at one particular observer time. The integration is complex because the pointwise mapping between the physical source plane and the $\Sigma$ surface is not known explicitly. Special care must be taken in the construction because in practice the $\Sigma$ surface may be composed of several disjoint pieces when the source motion is supersonic - exactly the case we are interested in. Figure 4 shows a typical evolution of the $\Sigma$ surface. Notice that in in the first few time steps each position on the $\Sigma$ surface is single valued (i.e., a point only occurs at one azimuthal position), but later the leading edge and eventually the entire outer portion of the surface becomes triple valued (i.e., point near the tip of the grid may occur at three azimuthal positions).

The marching cubes method begins constructing the $\Sigma$ surface by choosing the source time and computing the corresponding observer time and integrand value at each grid point. If the observer times are computed and stored for each desired source time, the discrete computational data become a three-dimensional array; two computational indices parameterize the surface spatially and a third index accounts for the source time. In this three-dimensional computational space, isosurfaces of observer time $t$ are, by definition, distinct realizations of the $\Sigma$ surface. The extension of the marching-cubes algorithm for surface integration [19] determines how the surface intersects a logical cube in the three-dimensional computational grid, computes the contribution to the integral from that portion of the surface, and then moves (or marches) to the next cube. The topology of the surface within a single cube can be determined uniquely by examining the function value (observer time in this case) at each of the cube vertices and comparing this value to the desired surface value. A table lookup is then used to determine the exact topology of the surface in the current cube. The surface is formed by a set of triangular panels that have vertices on the edges of the cubes. The value of the surface integral over each triangle is approximated as the average integrand value of 

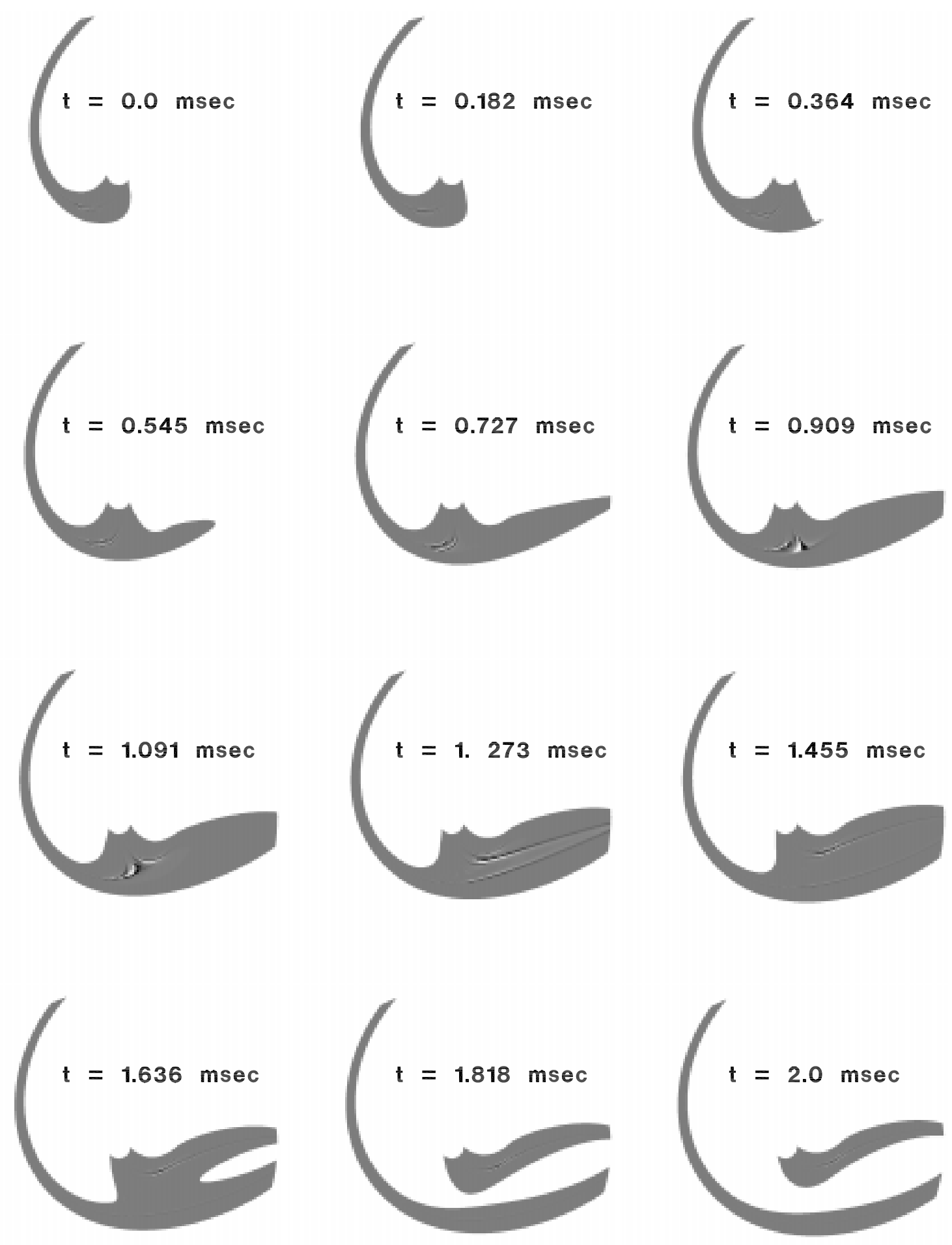

Figure 4. Evolution of the $\Sigma$-surface geometry and integrand strength distribution $\hat{r}_{i} \hat{r}_{j} L_{\tau}^{2} Q_{i j} / r$ a function of observer time. The observer is located 3.09R from the rotor hub and the rotor tip velocity is $M_{H}=0.95$. 
the triangle vertices multiplied by the triangle area. Linear interpolation is used to determine the integrand values at the triangle vertices based on the previously computed value at the cube vertices. The marching cubes algorithm is generic - the only difference in computing thickness, loading, or quadrupole noise is the value of the integrand computed at each grid vertex. (For more detail on the marching cubes algorithm, see references 19 and 20.)

The version of the marching-cubes algorithm used for the calculations in this paper was adaptive- that is the integral value for a single cube was compared to the value obtained using the 8 subcubes obtained by bisecting each of the sides of the original cube. If the difference between the integral values was greater than a specified tolerance, each of the subcubes were also subdivided in a recursive manner. Six levels of recursion were allowed in the computations for this paper. When a cube was divided, the quadrupole source strength at the new vertices was computed using linear interpolation, but the observer time and source location were computed without approximation. Finally the integrand values were computed anew at each of the new vertex locations.

For simplicity, the current WOPWOP2+ code only implements formulation Q2 for a hovering rotor. For a hovering rotor, $L_{\tau} Q_{i j}$ and $L_{\tau}^{2} Q_{i j}$ can be written in the form

$$
L_{\tau} Q_{i j}=-(\boldsymbol{\omega} \times \boldsymbol{\eta}) \cdot \nabla_{\eta} Q_{i j}=-\omega \eta \frac{\partial Q_{i j}}{\partial \eta_{1}}
$$

and

$$
L_{\tau}^{2} Q_{i j}=(\boldsymbol{\omega} \times \boldsymbol{\eta}) \cdot \nabla_{\boldsymbol{\eta}}\left((\boldsymbol{\omega} \times \boldsymbol{\eta}) \cdot \nabla_{\boldsymbol{\eta}} Q_{i j}\right)=\omega^{2} \eta^{2} \frac{\partial^{2} Q_{i j}}{\partial \eta_{1}^{2}}
$$

where $\partial / \partial \eta_{1}$ is the directional derivative in the azimuthal direction and $\eta=|\boldsymbol{\eta}|$. Equation (18) is implemented numerically in WOPWOP2+ with a second-order accurate central-difference operators.

\section{NUMERICAL RESULTS}

In this section we first make a comparison of the acoustic pressure signatures of a hovering model rotor from WOPWOP + and WOPWOP2+ which use formulations Q1A and Q2, respectively. Then, we present the comparison of the predicted and measured acoustic pressure signatures for the same hovering model rotor at four different tip Mach numbers. At the three highest tip Mach numbers, the phenomenon of delocalization occurs and WOPWOP2+ must be used for HSI noise prediction if the contribution of the supersonic quadrupoles is to be included in the prediction. The remaining analysis examines the role of the supersonic quadrupoles and the robustness of the formulation.

A model-scale rotor test conducted by Boxwell et al. in 1978 [25] and repeated later by Purcell in 1988 [26] is used for comparison. The measured data was for nonlifting hovering rotor generating HSI noise. The rotor was a $1 / 7$ th scale UH-1H main rotor with straight untwisted blades and NACA 0012 airfoil section. The rotor radius $R$ was $1.045 \mathrm{~m}$ with a chord of $7.62 \mathrm{~cm}$. The measured data reported here are all from a microphone in the rotor plane and at $3.09 R$ from the rotor tip. The Euler solutions utilized as input in this numerical work were provided by Baeder and are described in references 5 and 24 . The Euler solutions are also used for direct comparison with the acoustic prediction when experimental data is unavailable.

\subsection{COMPARISON OF FORMULATIONS}

Figure 5 shows a comparison of the predicted acoustic pressure signatures from WOPWOP + (formulation $\mathrm{Q} 1 \mathrm{~A}$ ) and WOPWOP2+ (formulation Q2) at tip Mach number 0.925. The thickness and loading, quadrupole, and total acoustic pressure time histories predicted by each of the codes are also shown in this figure. Although the supersonic quadrupoles are important in prediction of the acoustic pressure signature because delocalization occurs at this operating condition, WOPWOP + can only handle subsonic quadrupole sources; therefore, we have only used the subsonic quadrupole sources in both predictions for this comparison. We have utilized the marching cubes approach to construct the $\Sigma$ surface (influence surface) of the rotor blade and the quadrupole source surface in WOPWOP2+. A good agreement in this comparison proves two points. First, it will tell us that the construction of the $\Sigma$ surface is correct in WOPWOP2+. Second, the two formulations Q1A and Q2 are equivalent. Both these points are evident in figure 5. This figure also shows that the individual components due to thickness and loading and the quadrupole sources as well as the 

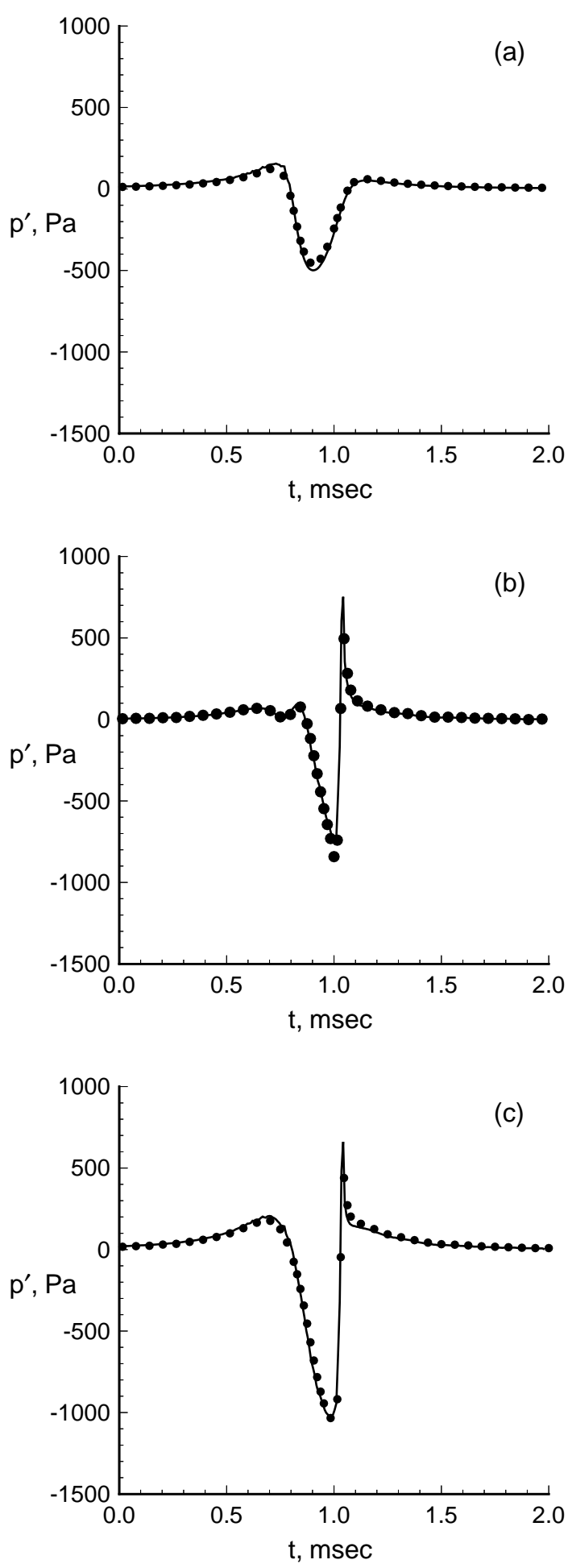

Figure 5. Comparison of WOPWOP + prediction $(\bullet)$ with subsonic WOPWOP2+ prediction $(-)$ for a $\mathrm{UH}-1 \mathrm{H}$ hovering rotor, $M_{H}=0.925$. Quadrupole grid extends $0.075 R$ beyond rotor tip. (a) thickness and loading components; (b) quadrupole component; (c) total acoustic pressure. 
total acoustic pressure signatures from the two codes agree well. Thus, we have established some confidence in using WOPWOP2 + for prediction of HSI noise.

\subsection{COMPARISON WITH MEASURED DATA}

We now present HSI noise calculations for tip Mach numbers $0.88,0.9,0.925$, and 0.95 in figure 6 . The quadrupole grid extends $1.86 R$ beyond the blade tip for all the WOPWOP $2+$ calculations shown in figure 6 . For comparison, we have also shown the WOPWOP + signature which includes quadrupole sources up to the sonic circle. It is seen that the agreement of the WOPWOP2+ signature with the measured data is excellent and better than that of WOPWOP + for each case. We have, thus, demonstrated the ability to predict the noise from supersonic quadrupoles in the case of delocalized shocks and the resulting improvements in the overall shape and level of the acoustic pressure.

For the more intense cases $\left(M_{H}>0.90\right)$, the agreement of the WOPWOP2+ prediction with the measured acoustic pressure signature is not fully satisfactory because the WOPWOP2+ prediction overpredicts the negative peak pressure. This is apparent in figure 6 for the times between the WOPWOP + and the WOPWOP2+ shock locations. A closer examination of the $\Sigma$ surface and the quadrupole integrand strength distribution for this time range, shown in figure 7, reveals that this time range is precisely when the region of the strongest quadrupole integrand strength is is bifurcating (i.e. transitioning from subsonic to supersonic). Notice in particular that the area of the highest and lowest values (lightest and darkest, respectively) change dramatically during this time range. Hence relatively small errors in the peak values of $L_{\tau}^{2} Q_{i j}$ can result in amplified error. To reduce the sensitivity of the acoustic predictions to this type of error, we utilized a 7 point, moving-window-average smoothing in the chordwise direction of the quadrupole source strength. This smoothing had only a small effect on the acoustic signal away from the shock, but was useful to reduce the error previously noted. Figure 8 is a representative comparison of the smoothed and unsmoothed acoustic predictions. (Note smoothing was used in all of the computations for this paper except the $M_{H}=0.88$ case where the shock strength did not seem to warrant smoothing.)

It is well known that the quadrupole accounts for the nonlinear propagation effects caused by the finite particle velocity and the variation of sound speed in the physical problem. The calculations shown in figure 6 seems to indicate that the primary role of the supersonic quadrupoles is to increase the pulse width of intense propagating waves. The width of the main pulse of the signatures predicted by WOPWOP2+ agrees very well with the measured signature. Even for the $M_{H}=0.88$ case, which is not delocalized, the supersonic quadrupoles improve the agreement by increasing the width and amplitude of the acoustic signal. For the delocalized cases, the supersonic quadrupoles also decrease the slope, and thus improve the agreement, of the triangular shape proceeding the rapid increase in acoustic pressure. Figure 9 shows the effect of the extent of the quadrupole grid for the particularly intense $M_{H}=0.95$ case. Three separate computations were made with the quadrupole source grid extending beyond the rotor tip $0.05 R, 0.33 R$, and $1.86 R$, respectively. The quadrupole grid is shown in figure $9 \mathrm{a}$ with the three grid extents indicated. The first WOPWOP2+ computation, $0.05 R$ beyond the rotor tip, is essentially identical to the WOPWOP + calculation shown in figure $6 \mathrm{~d}$. Notice that the prediction for the largest grid extent, $1.86 R$ beyond the rotor tip, agrees very well with the data in both waveform width and shape. These computations suggest two things: even though the quadrupole correctly predicts the nonlinear propagation (i.e., the widening and changing of the waveform shape), it is probably an inefficient tool for predicting the nonlinear propagation because an accurate CFD computation must proceed the quadrupole prediction; and it seems likely that the nonlinear propagation could be determined more appropriately by another method starting with the acoustic signal somewhat closer to the rotor.

\subsection{FORMULATION ROBUSTNESS}

In this section we wish to demonstrate the robustness of the method by performing predictions which violate some of the assumptions leading to formulation Q2. First we predict the noise for out-of-plane observer and compare the acoustic pressure with an Euler solution. Secondly, we predict the noise for a very near-field observer.

The acoustic pressure was predicted using WOPWOP + and WOPWOP2+ at two observer locations directly below the an in-plane observer at $3.09 \mathrm{R}$ from the rotor hub. These observers are 10 and 20 deg below the rotor plane, respectively. In figure 10 we show these prediction for the $M_{H}=0.9$ case. No measured data are available at these observer locations, therefore, we have interpolated the Euler solutions [5] used as input. It is seen that the two sets of calculations agree fairly well with each other, but the WOPWOP2+ result 

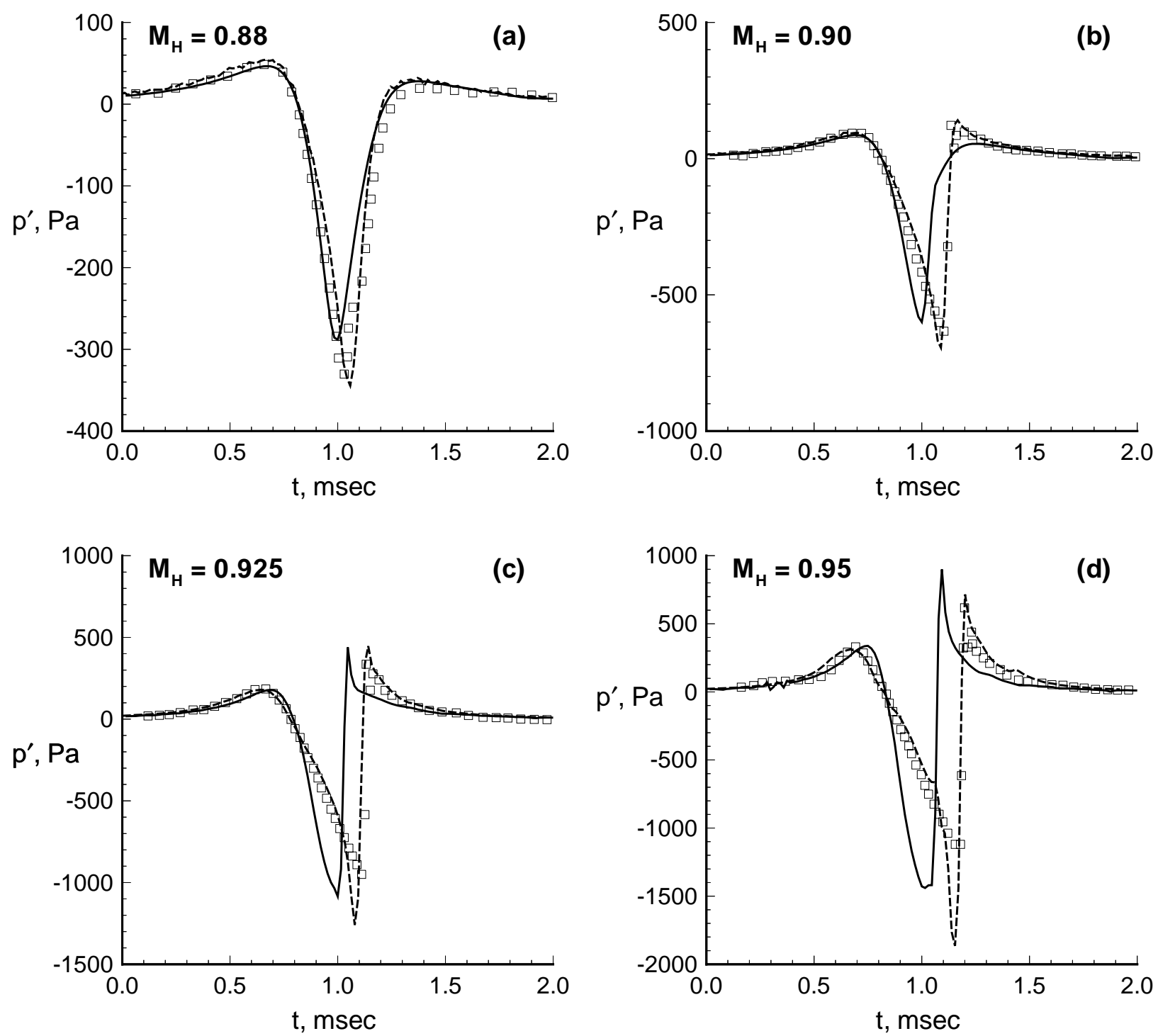

Figure 6. Comparison of WOPWOP $+(-)$ and WOPWOP2+ $(---)$ predicted acoustic pressure with experimental data [26] () for hovering model UH-1H rotor. Quadrupole grid in WOPWOP+ prediction extended almost to sonic circle and in WOPWOP2+ predictions extended $1.86 R$ beyond the rotor tip. (a) $M_{H}=0.88$; (b) $M_{H}=0.90$; (c) $M_{H}=0.925$; (d) $M_{H}=0.95$. 

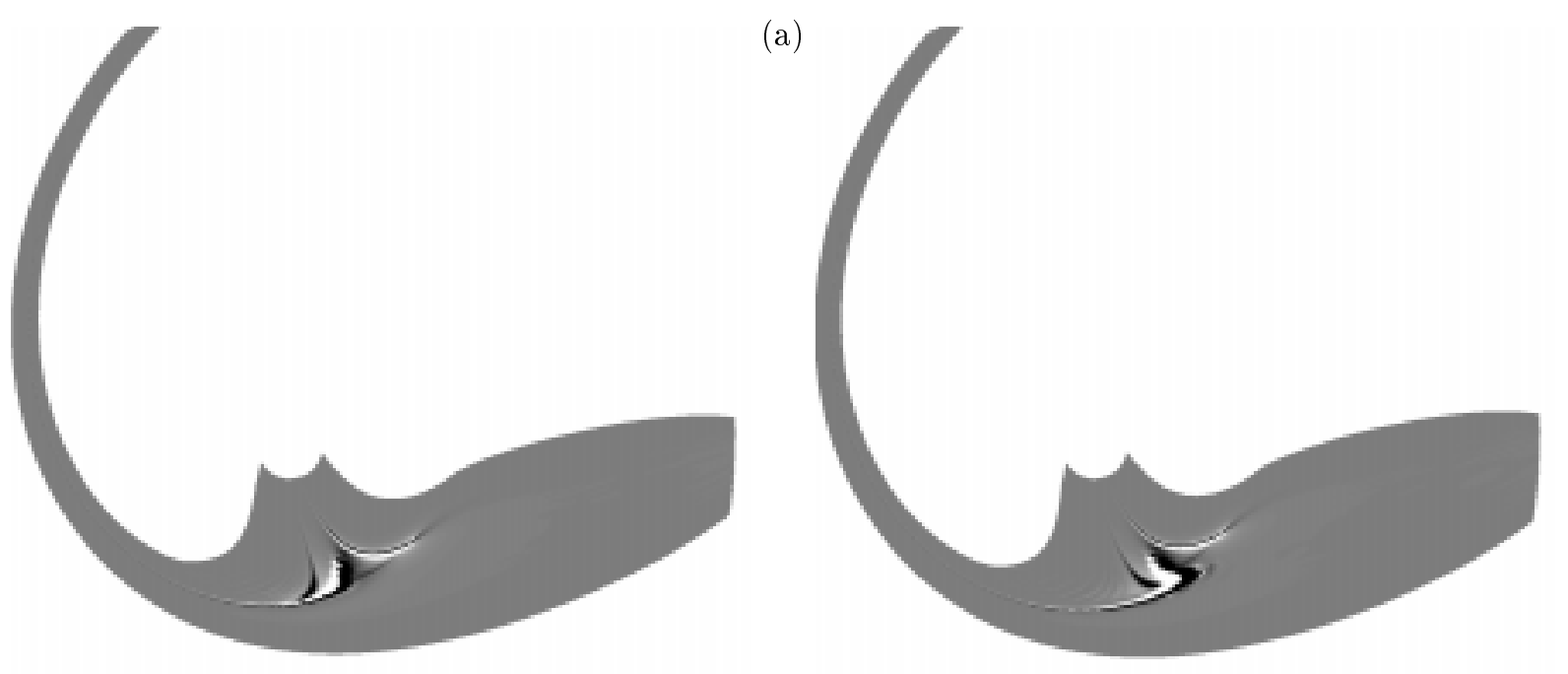

(b)
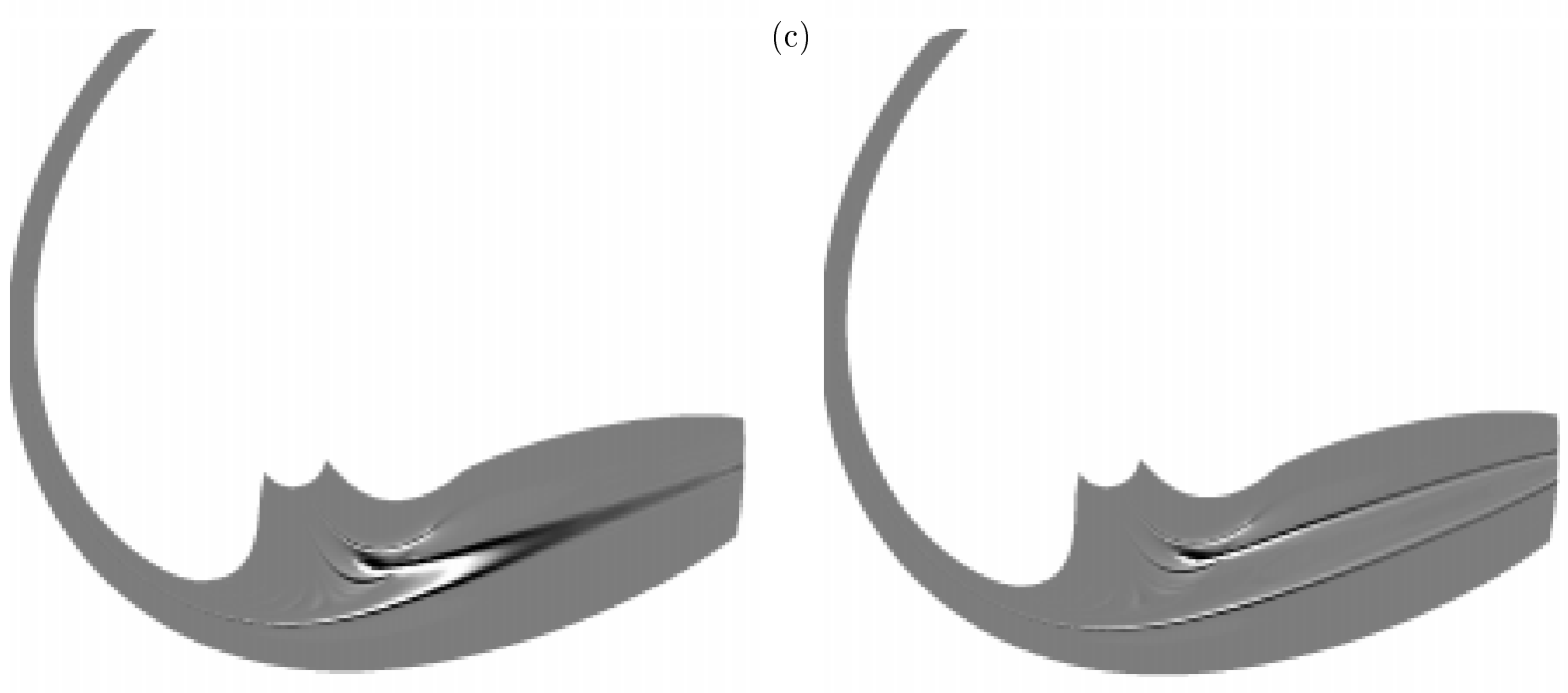

(d)

Figure 7. Evolution of quadrupole integrand strength distribution $\hat{r}_{i} \hat{r}_{j} L_{\tau}^{2} Q_{i j} / r$ on the emission surface as a function of observer time for times near the peak negative acoustic pressure. Dark and light shading indicates negative and positive values of $\hat{r}_{i} \hat{r}_{j} L_{\tau}^{2} Q_{i j} / r$ respectively. Note the extremely rapid change in the contours area as the source region bifurcates.(a) $t=1.050 \mathrm{msec}$; (b) $t=1.117 \mathrm{msec}$; (c) $t=1.183 \mathrm{msec}$; (d) $t=1.250$ msec. 


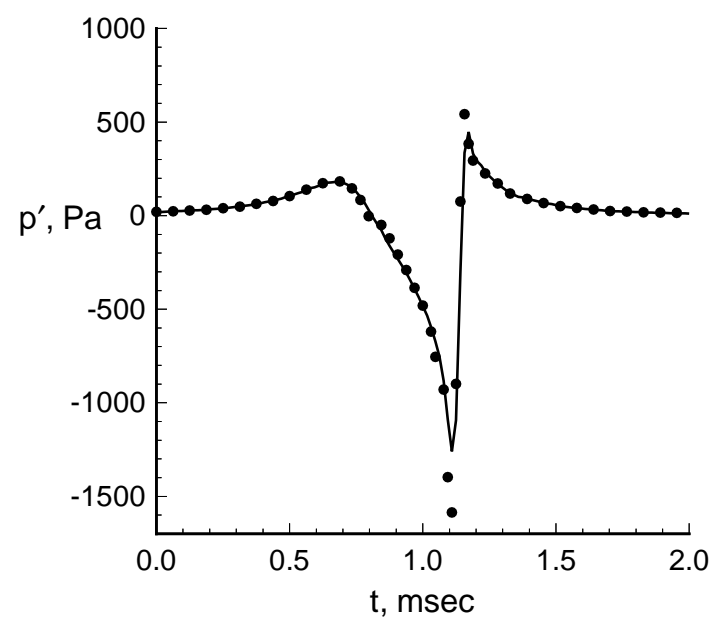

Figure 8. Comparison of WOPWOP2+ prediction with unsmoothed $Q_{i j}$ input data $(\bullet)$ with the prediction made using $Q_{i j}$ smoothing (-). A 7-point moving-window average smoothing was applied in the chordwise direction for a UH-1H hovering rotor, $M_{H}=0.925$.

characterizes the waveform width and asymmetry slightly better for the 10 deg down observer. Furthermore, as expected, the peak negative value of the main pulse of the acoustic pressure reduces with increasing observer angle. When the observers is $20 \mathrm{deg}$ below the rotor plane the agreement is good for both methods, but the quadrupole contribution is relatively small. The chordwise smoothing of the quadrupole source strength in the WOPWOP2 + computation is believed to be responsible for the lower amplitude in the $10 \mathrm{deg}$ down prediction; since the quadrupole contribution is small in the 20 deg down case the effect of smoothing is not readily noticeable.

In a second comparison, shown in figure 11, we have predicted the acoustic pressure with WOPWOP $2+$ at an in-plane observer $1.094 R$ from the rotor hub. This observer is just inside the sonic circle and is less than two chordlengths from the rotor tip at the closest distance. The quadrupole grid extends almost to the observer. WOPWOP + was unable to determine the retarded time satisfactorily for this severe test case because the root finding algorithm exceeded the number of iterations allowed. The WOPWOP2+ prediction slightly underpredicts the Euler solution; nevertheless, the comparison is extremely good at this very nearfield location. In particular, the WOPWOP2+ prediction agrees perfectly with the Euler data both before the negative peak and for the curved part of the signal at the top of the shock-like structure after the negative peak. The far-field and near-field quadrupole terms (terms with $1 / r$ dependence and terms with $1 / r^{2}$ and $1 / r^{3}$ dependence, respectively) from equation (7) are also shown in figure 11 . Clearly the near-field quadrupole terms - usually neglected by other researchers - contribute significantly to the correct prediction of waveform shape at this close distance. Figure 11 demonstrates the importance of keeping all of the terms so that the acoustic prediction can be compared directly with CFD [16]. Both figures 10 and 11 demonstrate the robustness of formulation Q2.

\section{CONCLUDING REMARKS}

We have presented a new quadrupole noise prediction method based on a new analytic result, called formulation Q2, valid for both subsonic and supersonic quadrupole sources. The new formulation is very simple and without any singularity. The procedure for implementation of the result is discussed in the paper. This new code is called WOPWOP2+. We have demonstrated that formulation Q2 is equivalent to formulation Q1A of Brentner used in WOPWOP + for subsonic quadrupole sources. By order of magnitude study of the formulation Q2 far-field integrand, we have shown that the shock surfaces and the stagnation flow at the leading edge of the blade are regions of high source intensity. We have shown that for rotors operating at high tip Mach numbers - before and after delocalization - the new formulation predicts acoustic pressure signatures which agree well with the experimental data in both the shape and the level of the main 
(a)
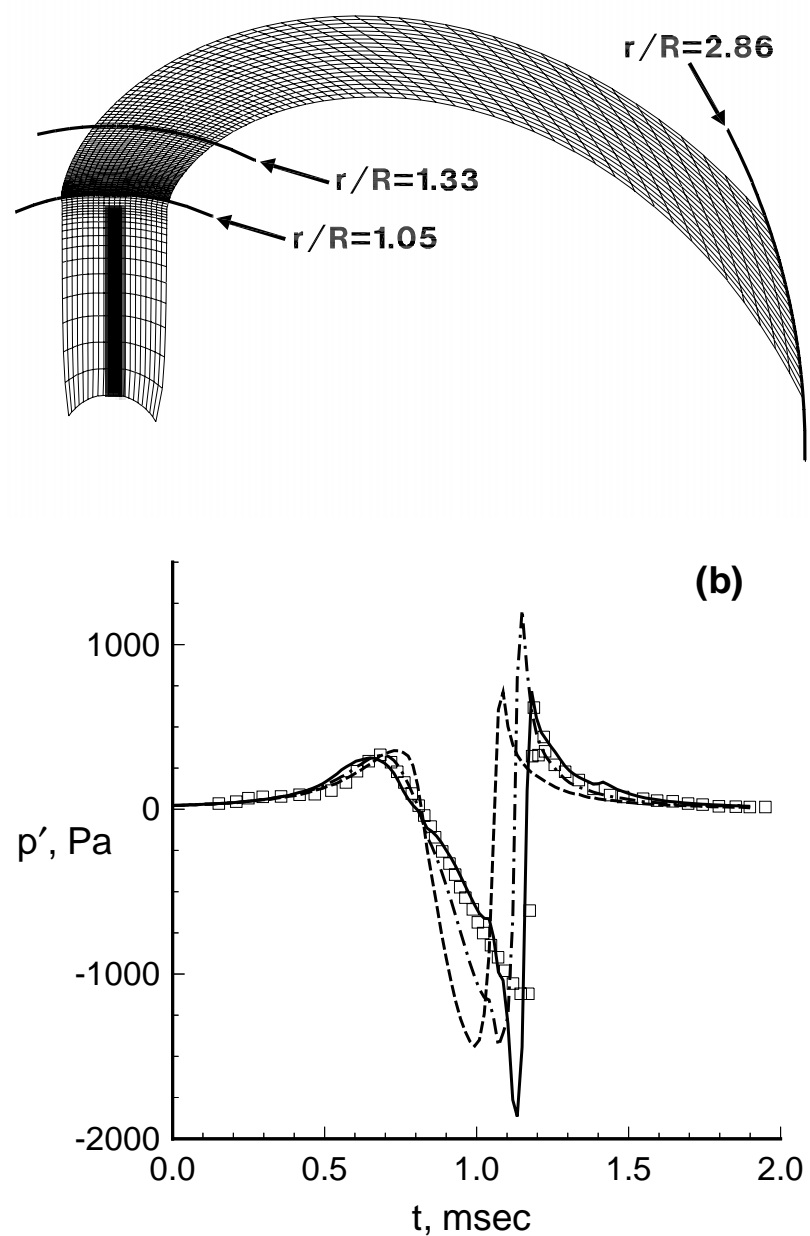

Figure 9. Effect of quadrupole grid extent shown for hovering UH-1H rotor, $M_{H}=0.95$. (a) quadrupole grid (every 10th grid line in chordwise direction shown); (b) predicted acoustic pressure for three quadrupole grid extents:,$--- 0.05 R$ beyond rotor tip (subsonic); - -, $0.33 R$ beyond rotor tip;,$- 1.86 R$ beyond rotor tip; $\mathbf{c}$ experimental data [26]. 

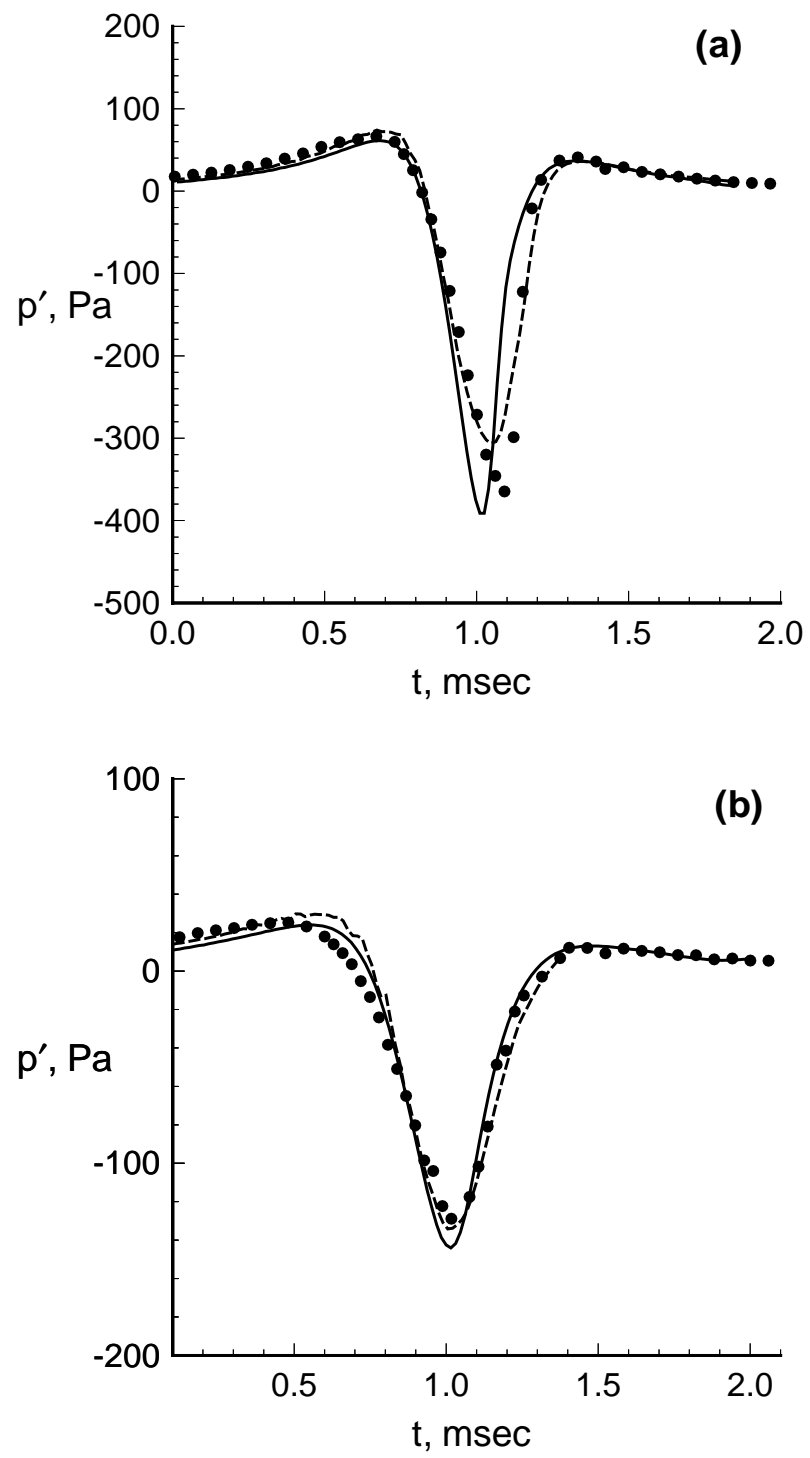

Figure 10. Comparison of WOPWOP $+(-)$, WOPWOP2+ $(-\boldsymbol{-}-)$, and Euler [5,24] $(\bullet)$ predicted acoustic pressures for hovering model UH-1H rotor, $M_{H}=0.90$. (a) 10 deg below rotor plane; (b) 20 deg below rotor plane. 


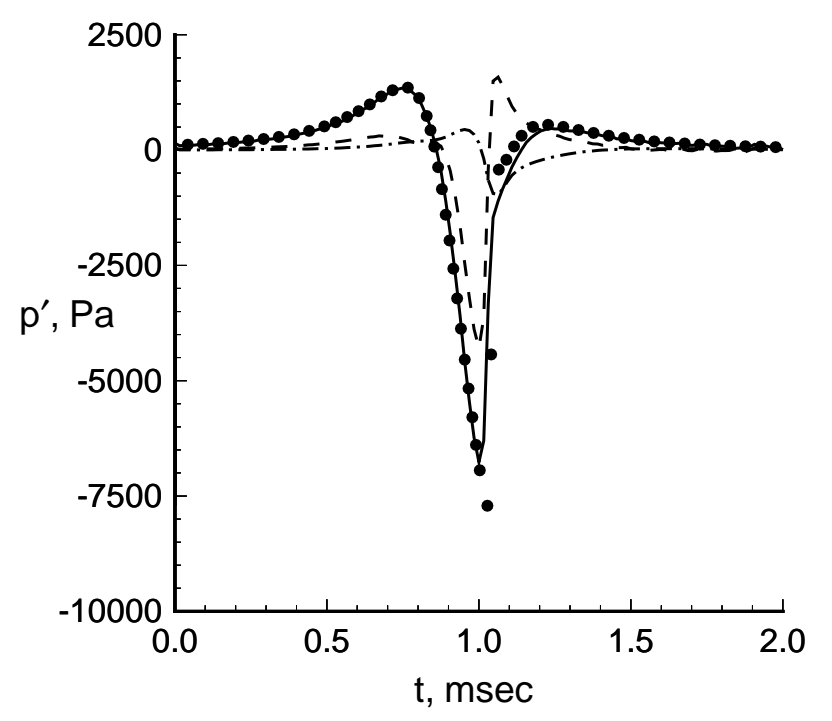

Figure 11. Near-field noise prediction at in-plane observer located $1.094 R$ from rotor hub for hovering model UH-1H rotor, $M_{H}=0.90$ with quadrupole grid $0.0935 R$ beyond rotor tip. - - - , far-field quadrupole component; -- - , near-field quadrupole components; - - WOPWOP2+ total acoustic pressure; •, Euler $[5,24]$ total acoustic pressure.

pulse of the signature. We have also shown that the supersonic quadrupoles widen and modify the shape of the waveform. A new and robust option is now available for prediction of HSI noise of helicopter rotors based on formulation Q2.

\section{REFERENCES}

1. Y. H. Yu, F. X. Caradonna and F. H. Schmitz 1978. Fourth European Rotorcraft and Powered Lift Aircraft Forum. (Paper 58.) The influence of the transonic flow field on high-speed helicopter impulsive noise.

2. D. B. HAnson and M. R. Fink 1979 Journal of Sound and Vibration 62(1), 19-38. The importance of quadrupole sources in prediction of transonic tip speed propeller noise.

3. K. J. Schultz and W. R. Splettstoesser 1987. American Helicopter Society 43rd Annual Forum. Prediction of helicopter rotor impulsive noise using measured blade pressure.

4. J. Prieur 1986 AIAA Paper 86-1901. Calculation of transonic rotor noise using a frequency domain formulation.

5. J. D. Baeder 1991. American Helicopter Society/Royal Aeronautical Society International Technical Specialists' Meeting on Rotorcraft Acoustics and Rotor Fluid Dynamics. Euler solutions to nonlinear acoustics of non-lifting rotor blades.

6. K. S. Brentner and P. C. Holland 1997 Journal of the American Helicopter Society 42(2), 172-181. An efficient and robust method for computing quadrupole noise.

7. K. S. Brentner 1997 Journal of Sound and Vibration 203(1). An efficient and robust method for predicting helicopter rotor high-speed impulsive noise.

8. P. Di Francescantonio 1997 Journal of Sound and Vibration 202(4), 491-509. A new boundary integral formulation for the prediction of sound radiation.

9. K. S. Brentner and F. Farassat 1998 AIAA Journal 36(8), 1379-1386. An analytical comparison of the acoustic analogy and kirchhoff forumulation for moving surfaces. 
10. K. S. Brentner, A. S. Lyrintzis and E. K. Koutsavdis 1997 Journal of Aircraft 34(4), 531-538. Comparison of computational aeroacoustic prediction methods for transonic rotor noise prediction.

11. R. C. Strawn and R. Biswas 1995 Journal of the American Helicopter Society 40(3), 66-72. Computation of helicopter rotor noise in forward flight.

12. P. Di Francescantonio 1996 AIAA Paper 96-1708. A supersonically moving kirchhoff surface method for delocalized high speed rotor noise prediction.

13. G. Rahier and J. Prieur 1997. American Helicopter Society 53rd Annual Forum. An efficient kirchhoff integeration method for rotor noise prediction starting indifferently from subsonically or supersonically rotating meshes.

14. S. IANniello 1998 AIAA 98-2377. Quadrupole noise predictions through the FW-H equation.

15. S. IAnNiEllo 1998 AIAA 98-2378. An algorithm to integrate the Ffowcs Williams-Hawings equation on a rotating, supersonic domain.

16. F. Farassat and K. S. Brentner 1988 Journal of the American Helicopter Society 33(1), 29-36. The uses and abuses of the acoustic analogy in helicopter rotor noise prediction.

17. F. FARASSAT 1975 NASA TR R-451. Theory of noise generation from moving bodies with an application to helicopter rotors.

18. F. Farassat 1994 NASA TP 3428. (See corrected version, April 1996. Available online at ftp://techreports.larc.nasa.gov/pub/techreports/larc/94/tp3428.ps.Z directly.) Introduction to generalized functions with applications in aerodynamics and aeroacoustics.

19. K. S. Brentner 1994 Proceedings of the IMACS 14th World Congress on Computational and Applied Mathematics, 592-595. A new algorithm for computing acoustic integrals.

20. W. E. Lorensen and H. E. Cline 1987 Computer Graphics 21(4), 163-169. Marching cubes: A high resolution 3d surface construction algorithm.

21. F. Farassat 1987 AIAA Paper 87-2675. Quadrupole source in prediction of noise of rotating blades-a new source description.

22. F. Farassat, Y.-J. Lee, H. Tadghighi and R. Holz 1991. American Helicopter Society/Royal Aeronautical Society International Technical Specialists' Meeting on Rotorcraft Acoustics and Rotor Fluid Dynamics. High-speed helicopter rotor noise - shock waves as a potent source of sound.

23. H. Tadghighi, R. Holz, F. Farassat and Y. J. Lee 1991. American Helicopter Society 47th Annual Forum. Development of a shock noise prediction code for high-speed helicopters.

24. J. D. Baeder, J. M. Gallman and Y. H. Yu 1997 Journal of the American Helicopter Society 42(1), 39-53. A computational study of aeroacoustics of rotors in hover.

25. D. A. Boxwell, Y. H. Yu and F. H. Schmitz 1979 Vertica 3(1), 35-45. (Also in NASA CP-2052, 1978.) Hovering impulsive noise: Some measured and calculated results.

26. T. W. Purcell 1988. Fourteenth European Rotorcraft Forum. (Paper 2.) CFD and transonic helicopter sound. 